\title{
Chaotic microlasers based on dynamical localization
}

\author{
Viktor A. Podolskiy* ${ }^{\star \ddagger}$, Evgenii Narimanov* ${ }^{\star \dagger}$ Wei Fang ${ }^{\S}$, and Hui Cao ${ }^{\dagger \S}$ \\ *Electrical Engineering Department, Princeton University, Princeton, NJ 08544; and §Department of Physics and Astronomy, Northwestern University, \\ Evanston, IL 60208
}

Edited by Marlan O. Scully, Texas A\&M University, College Station, TX, and approved June 10, 2004 (received for review April 20, 2004)

\begin{abstract}
We report the direct observation of lasing action from a dynamically localized mode in a microdisk resonator with rough boundary. In contrast to microlasers based on stable ray trajectories, the performance of our device is robust with respect to the boundary roughness and corresponding ray chaos, taking advantage of Anderson localization in angular momentum. The resonator design, although demonstrated here in GaAs-InAs microdisk laser, should be applicable to any lasers and sensors based on semiconductor or polymer materials.
\end{abstract}

$\mathbf{T}$ he resonator cavity is an essential part of any lasing device, providing the necessary energy accumulation and (coherent) feedback for the mode development. Dielectric microdisks and microcylinders are among the most widely used resonator cavities in modern microlasers and microsensors (1-3). The performance of such resonators is usually related to their internal classical ray dynamics. Here we show that quantum interference present in any wave-optical system may dramatically affect the microlaser behavior, and demonstrate the effect of dynamical localization (DL) (4-8) on the formation of the lasing mode. We report the direct observation of DL in optical system, and demonstrate that the performance of the DL-based resonator is robust with respect to boundary roughness, a natural limiting factor of ray-based devices.

In a circular microdisk, classical rays form a series of whispering-gallery (WG) trajectories with conserved angular momentum and, correspondingly, a conserved angle of incidence (Fig. 1). Because the refractive escape is only possible for the trajectories with the angle of incidence $\chi$ below the critical angle $\chi_{\mathrm{c}}=\arcsin (1 / n)(n$ being the refractive index of the dielectric), the rays with the angle of incidence $\chi>\chi_{\mathrm{c}}$ (Fig. 1) are trapped inside the dielectric microdisk by the total internal reflection. The quantum modes localized on these quasiperiodic trajectories can therefore leave the resonator only via evanescent (tunneling) escape, so they usually have extremely long lifetimes and correspondingly low lasing thresholds.

Even a small roughness of the resonator boundary can destroy its rotational symmetry and the stability of WG trajectories, making the classical dynamics inside the cavity completely chaotic (Fig. 1). As a ray trajectory propagates in a chaotic microdisk, its angular momentum is changed in a quasirandom manner, until it reaches the region $\chi<\chi_{\mathrm{c}}$, when the ray may refractively escape from the system (Fig. 1). It is natural to assume that the modes inside such a rough cavity resemble classical trajectories and have small lifetimes. This assumption imposes severe limitations on the microlaser fabrication. As a result, state-of-the-art microfabrication technology is generally used to minimize the roughness of the microcavity boundary and prevent the ray chaos.

However, the wave dynamics inside the rough microdisks may substantially differ from the ray picture. The chaotic diffusion, which leads to the divergence of the initially close rays, may be strongly suppressed because of their destructive interference. Therefore, a wavepacket, initially localized in the angular momentum, may remain localized as it propagates inside the rough cavity. Because in this case the localization is related to dynamics of the wavepacket, this effect is referred to as the dynamical localization (4-6).
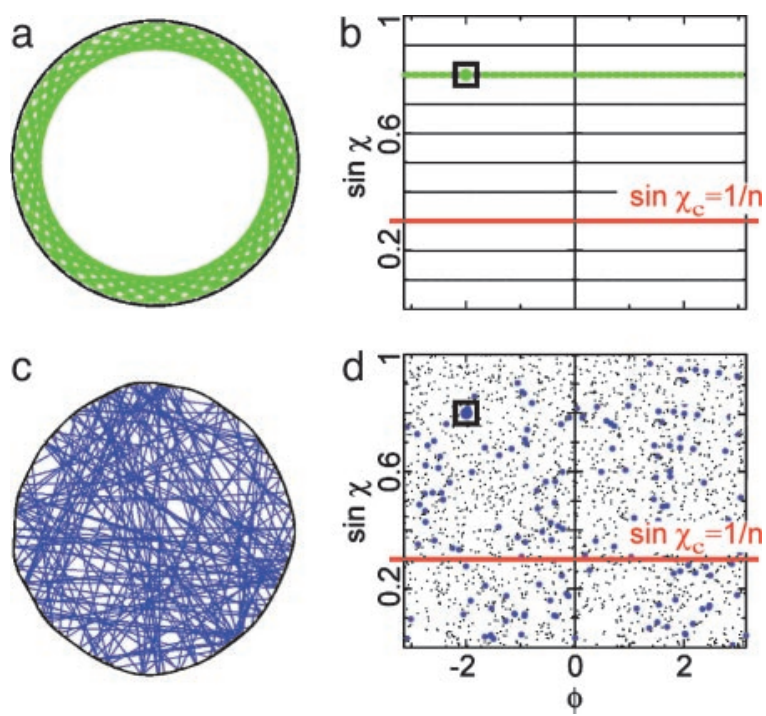

Fig. 1. Ray dynamics inside the microdisk resonator. (a) Ray trajectories inside the circular microdisk have WG structure. The conserved angular momentum of any given trajectory is due to the symmetry of the microdisk boundary. This fact is clearly seen in the Poincare surface of section (SOS) (b), consisting of a series of one-dimensional lines, each corresponding to an orbit with a fixed angle of incidence, $\chi$. The green dots correspond to the trajectory in $a$; the bold dot in the black square denotes the starting point. The trajectories with $\sin \chi>1 / n$, where $n$ is the refraction index of the cavity, are trapped by total internal refraction (red line corresponds to the experimental value $n=$ 3.25). (c) Introduction of roughness to the microcavity boundary destroys the stability of all WG orbits. Total ray chaos is illustrated through the system's SOS, shown in $d$. The single trajectory now explores all available phase space and may classically escape from the resonator when its $\sin \chi$ is below $1 / n$; the blue dots show the trajectory in $c$, which has the same initial conditions as in the trajectory in a (bold blue dot in the black square).

The phenomenon of DL can also be seen in the stationary solutions of the Maxwell equations. Namely, the wavefunctions inside the rough microcavities become localized in the angular momentum space (Fig. 2b). The real space structures of such dynamically localized modes are somewhat close to that of WG trajectories (Fig. $2 a$ ).

As a result, modes localized near the angular momentum corresponding to $\chi>\chi_{\mathrm{c}}$ might have long lifetimes comparable to those of WG modes in smooth circular cavity (Fig. 2). This, in turn, enables the development of microdisk lasers robust with respect to boundary roughness.

Our dielectric microlasers are made of a 200-nm-thick GaAs layer with a thin InAs quantum well in the middle serving as the gain medium. The microdisks are fabricated by optical lithog-

\footnotetext{
This paper was submitted directly (Track II) to the PNAS office. Abbreviations: DL, dynamical localization; WG, whispering-gallery.

†To whom correspondence may be addressed. E-mail: vpodolsk@physics.orst.edu, evgenii@princeton.edu, or h-cao@northwestern.edu.

¥Present address: Department of Physics, Oregon State University, Corvallis, OR 97331. C 2004 by The National Academy of Sciences of the USA
} 

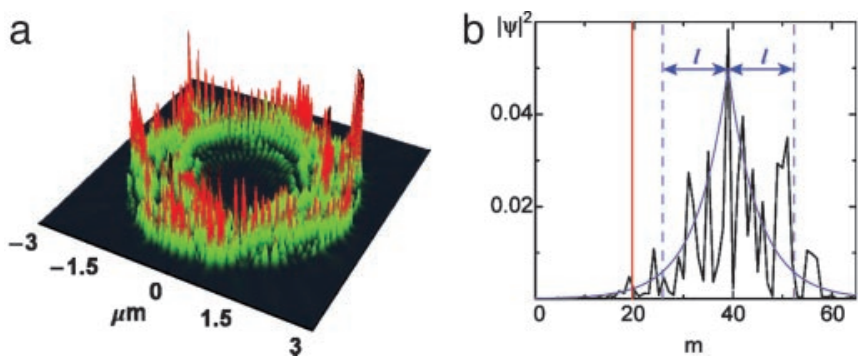

Fig. 2. The structure of the dynamically localized mode. (a) The intensity distribution of the dynamically localized mode is similar to regular WG trajectory; however, nontrivial interference patterns reveal strong deviation from the WG character. The angular momentum distribution of the mode (b) serves as a clear evidence of the DL with localization length, $I=12.4$. The lifetime of the mode is directly related to its angular momentum distribution and is determined by the absolute values of components with angular momenta, corresponding to an angle of incidence $\chi<\chi_{c}$ (red line corresponds to $\left.\chi_{c}\right)$. The Q-factor of the mode shown is $4.78 \times 10^{3}$.

raphy and two steps of wet etch (9). The disk diameter is close to $5 \mu \mathrm{m}$. As shown in Fig. 3, each disk is supported by a 500-nm-long $\mathrm{Al}_{0.7} \mathrm{Ga}_{0.3}$ pedestal. The top-view scanning electron microscope image reveals that the disk has a rough boundary (Fig. 3 Inset).

To study their lasing properties, the dielectric microcavities are cooled to $10 \mathrm{~K}$ in a cryostat and optically pumped by a mode-locked Ti-sapphire laser at $790 \mathrm{~nm}$. The pump beam is focused by an objective lens onto a single disk. The same lens collects the microcavity emission and sends it to a spectrometer.

The emission spectrum features a broadband amplified spontaneous emission and a number of distinct peaks that correspond to the cavity modes. When the pump power exceeds a threshold, the emission intensity from a single mode exhibits a sudden increase accompanied by a simultaneous decrease of the mode linewidth, indicating the onset of lasing oscillations. At high pumping, the microdisk shown in Fig. 3 exhibits lasing in several cavity modes (Fig. 4a). A single mode corresponding to $\lambda=855.5 \mathrm{~nm}$ is selected by a narrow band-pass filter. The onset of lasing oscillations in this mode is clearly seen in Fig. $4 b$. Fig. $4 c$ shows its near-field pattern, imaged by a charge-coupled device camera. Note that the intensity of the lasing mode is concentrated near the disk edge, in contrast with the uniform distribution of amplified spontaneous emission across the disk, measured through the bandpass filter tuned away from the cavity resonances. Such intensity localization near the disk edge is the evidence of the DL in the microlaser. a

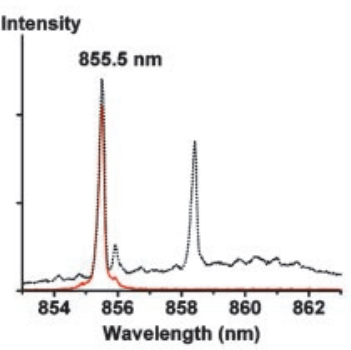

C

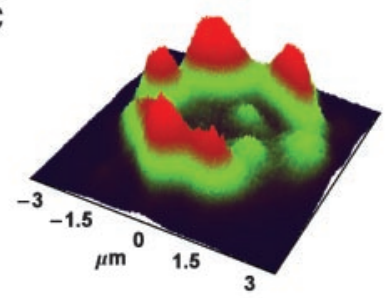

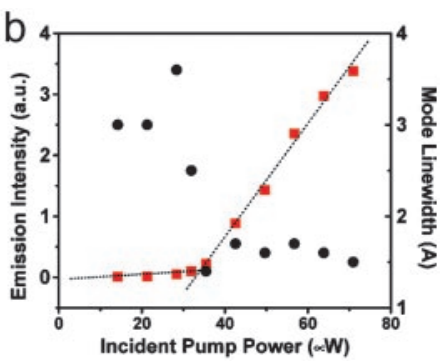

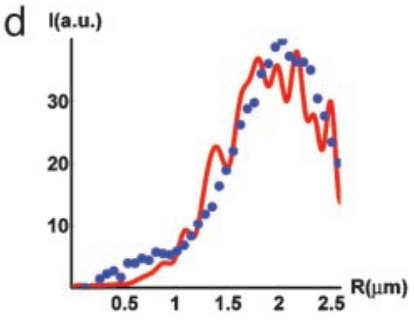

Fig. 4. Optical measurement of the microdisk in Fig. 3. The emission spectrum at the incident pump power of $44 \mu \mathrm{W}(a)$ clearly shows multimode lasing (black dotted line). A band-pass filter of $1 \mathrm{~nm}$ bandwidth selects a single lasing mode at $855.5 \mathrm{~nm}$ (red line). The onset of lasing oscillation in this mode $(b)$ is confirmed by the rapid increase of the emitted intensity as a function of the pump power (red squares) and simultaneous decrease of the linewidth (black circles) when the pump reaches the threshold of $35 \mu \mathrm{W}$. The near-field optical image of this lasing mode is shown in c. The agreement between the experiments and numerical simulations $(d)$ is shown through comparison of the radial distribution of the intensity of the mode in $c$ with subtracted constant amplified spontaneous emission background (blue dots) and the numerically simulated mode shown in Fig. 2 (red curve). Confinement of the lasing mode to the edge of the microdisk together with its angular momentum distribution shown in Fig. 2 is the evidence of its DL.

To confirm that the lasing mode is in fact dynamically localized, we use the scanning electron microscope image of the microdisk (Fig. 3a) to digitize its shape and numerically analyze its behavior. Our ray-tracing calculations confirm that the classical dynamics inside the cavity is completely chaotic (Fig. $1 c$ and $d$ ).

To numerically simulate the electromagnetic field distribution, we use the $S$-matrix formalism originally introduced in ref. 10, adopted to optical systems in ref. 11, and described in detail in ref. 12. In this approach, the electromagnetic field is represented as a series of cylindrical waves traveling to and from the center of the microdisk. These waves are related through the $S$-matrix, describing their reflection and refraction by the microdisk boundary. The eigenstates of the $S$-matrix are
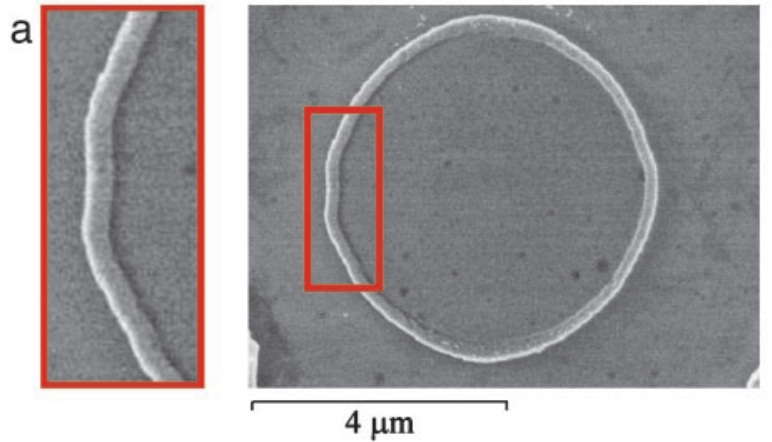

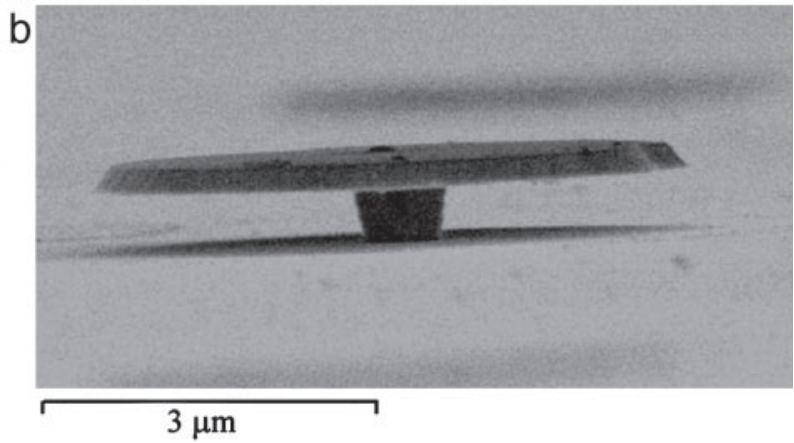

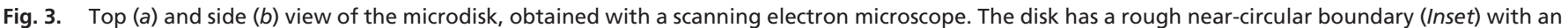

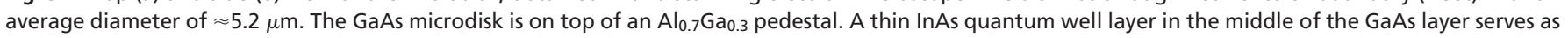
active medium. 
related to the solutions of the Maxwell equations inside the disk (11).

Using the $S$-matrix approach, we calculate the resonances in our system and identify the lasing mode shown in Fig. 2. Because a direct comparison of the simulated and measured intensity distributions is not possible due to the finite spatial resolution of the optical imaging setup, to avoid any fitting parameters, we compare the actual radial intensity distributions. Fig. $4 d$ shows that our numerical simulations well reproduce the experimental

1. Gmachl, C., Capasso, F., Narimanov, E. E., Nöckel, J. U., Stone, A. D., Faist, J., Sivco, D. L. \& Cho, A. Y. (1998) Science 280, 1556-1564.

2. Qian, S.-X., Snow, J., Tzeng, H.-M. \& Chang, R. K. (1986) Science 231, 486-488.

3. Nöckel, J. U. \& Stone, A. D. (1997) Nature 385, 45-47.

4. Casati, G., Chirikov, B. V., Ford, J. \& Izrailev, F. M. (1979) in Stochastic Behavior in Classical and Quantum Hamiltonian Systems, Lecture Notes in Physics, eds. Casati, G. \& Ford, J. (Springer, Berlin), Vol. 93, pp. 334-352.

5. Fishman, S., Grempel, D. R. \& Prange, R. E. (1982) Phys. Rev. Lett. 49, 509-512.

6. Frahm, K. M. \& Shepelyansky, D. L. (1997) Phys. Rev. Lett. 78, 1440-1443. data. The angular momentum and radial distribution of the lasing mode (Figs. 2 and 4) leave no doubt about its dynamically localized character.

This work was supported by National Science Foundation Grants DMR-0134736 and ECS-0400615 (to V.A.P. and E.N.) and National Science Foundation Materials Research Science and Engineering Center Program Grant DMR-0070697 (to W.F. and H.C.).

7. Sirko, L., Bauch, Sz., Hlushchuk, Y., Koch, P. M., Blumel, R., Barth, M., Kuhl, U. \& Stockmann, H.-J. (2000) Phys. Lett. A 266, 331-335.

8. Ringor, J., Szriftgiser, P., Carreau, J. C. \& Delande, D. (2000) Phys. Rev. Lett. 85, 2741-2744

9. Cao, H., Xu, J. Y., Xiang, W. H., Ma, Y., Chang, S.-H., Ho, S. T. \& Solomon, G. S. (2000) Appl. Phys. Lett. 76, 3519-3521.

10. Doron, E. \& Smilansky, U. (1992) Phys. Rev. Lett. 68, 1255-1258.

11. Starykh, O. A., Jacquod, P. R. J., Narimanov, E. E. \& Stone, A. D. (2000) Phys. Rev. E 62, 2078-2084.

12. Tureci, H. E., Schwefel, H. G. L., Jacquod, P. \& Stone, A. D. (2003) ArXiv: physics/0308016. 